\title{
A High Density of rRNA in the Generative Cells and Sperm Cells of Pollen Grains of Five Angiosperm Species
}

\author{
Chieko Saito ${ }^{1}$, Makoto Fujie ${ }^{2}$, Atsushi Sakai ${ }^{1}$, Noriko Nagata ${ }^{3}$, \\ Sachihiro Matsunaga ${ }^{1}$, Haruko Kuroiwa ${ }^{4}$ and Tsuneyoshi Kuroiwa ${ }^{1}$ \\ ${ }^{1}$ Department of Biological Sciences, School of Science, University of Tokyo, \\ Hongo 7-3-1, Tokyo 113-0033, Japan; E-mail: chiezo@biol.s.u-tokyo.ac.jp \\ ${ }^{2}$ Department of Molecular Biotechnology, Graduate School of Engineering, \\ Hiroshima University, Higashi-Hiroshima, 739-8527, Japan \\ ${ }^{3}$ Institute of Physical and Chemical Research (RIKEN), Wako 351-0198, Japan \\ ${ }^{4}$ Kyoritsu Woman's Junior College, Tokyo 101-0051, Japan
}

Accepted April 30, 1998

\begin{abstract}
Summary To examine whether cytoplasmic rRNA are present at high density in the cytoplasm of generative and sperm cells (generative/sperm cells) in pollen grains of various plant species, in situ hybridization of $18 \mathrm{~S} / 25 \mathrm{~S}$ rRNA was performed in thin sections of samples embedded in Technovit 7100 resin. The five plant species studied were Lilium longiflorum, Pharbitis nil, Silene latifolia, Pelargonium zonale and Plumbago auriculata. The timing and mode of pollen mitosis II (PM II) and the distribution of organelle nucleoids in generative/sperm cells differ in these species. In situ hybridization revealed that the density of rRNA was the same as or higher in generative/sperm cells than in vegetative cells, suggesting that the presence of rRNA in the generative/sperm cells at high density may be a general characteristic of mature pollen grains of various plant species.
\end{abstract}

Key words Generative cell, In situ hybridization, Pollen, rRNA, Sperm cell, Vegetative cell

In angiosperm pollen development, the asymmetric mitotic division of the haploid microspore (pollen mitosis one; PMI) results in the formation of two dimorphic cells, the vegetative cell and the generative cell, with very different fates (see reviews, Horvitz and Herskowitz 1992, Bedinger 1992, McCormick 1993, Tanaka 1997). The smaller generative cell produces two sperm cells (male gametes) after pollen mitosis two (PM II), whereas the larger vegetative cell, which constitutes the bulk of the pollen grain, produces an elongated pollen tube (a gametophytic cell) to deliver the male gametes to the embryo sac. The generative and sperm cells (generative/sperm cells) are entirely enclosed within the vegetative cell. The generative/sperm cells have very little cytoplasm, sparse nuclear pores (LaFountain Jr. and LaFountain 1973, Wagner et al. 1990) and condensed nuclear chromatin (Terasaka and Tanaka 1974, Twell et al. 1993, Saito et al. 1997). These characteristics suggest that the generative/sperm cells have lower transcriptional activity.

In a previous study, we demonstrated that the generative cells in pollen grains of Nicotiana tabacum contained certain amount of RNA, including rRNA, in their cytoplasm, although they had highly-condensed nuclear chromatin (Saito et al. 1997, 1998). In N. tabacum, however, the amount of cytoplasm in a generative cell is quite small, which made it difficult to demonstrate visually the difference in the density of rRNA between the generative cell and the vegetative cell in N. tabacum pollen. Then we demonstrated it separately by quantifying the density of rRNA in the generative and vegetative cells by microphotometry. To solve this difficulty, and to examine if rRNA are generally found at high density in the generative/sperm cells of various plant species.

The five plant species chosen for this study were Lilium longiglorum, Pharbitis nil, Silene latifolia, Pelargonium zonale and Plumbago auriculata. The timing and mode of PM II differ in these species. The mature pollen of L. longiflorum and $P$. nil is bicellular and the generative cell divides 
into two sperm cells after germination of the pollen tube, as in N.tabacum. However, the generative cells of L. longiflorum and $P$. nil have relatively more cytoplasm than N. tabacum, so that observation is easier. In contrast, the mature pollen of $S$. latifolia, P. zonale and P. auriculata is tricellular. In these plants, PM II occurs in the pollen grains before pollen tube germination. PM II is apparently symmetric in S. latifolia and $P$. zonale, while an asymmetric distribution of plastids and mitochondria occurs at PM II in P. auriculata. Because of the asymmetric distribution, one of the sperm cells in a mature pollen grain of $P$. auriculata contains most of the plastids, while the other contains most of the mitochondria (Saito et al. manuscript in preparation). This has also been reported in Plumbago zeylanica (Russell 1984, Sodmergen et al. 1995, Russell et al. 1996).

Angiosperms have been classified into two types, according to the behavior of the organelle nucleoids (or DNA) in the generative/sperm cells during the formation of pollen grains. This categorizing corresponds well to the classification based on the pattern of cytoplasmic inheritance (Kuroiwa 1991, Mogensen 1996). One type is characterized by the disappearance of organelle nucleoids in the generative/sperm cells, while the other is characterized by the presence of organelle nucleoids in generative/sperm cells. The first type shows maternal inheritance of the organelles, while the second shows biparental or paternal inheritance. The five species studied include both types. $N$. tabacum, L. longiflorum and S. latifolia are classified in the first group, whereas P. nil, $P$. zonale and $P$. auriculata are classified in the second.

In this study, in situ hybridization of cytoplasmic 18S/25S rRNA was performed in the pollen grains of five angiosperm species. The results demonstrated that the density of rRNA in the generative/sperm cells was similar or higher than that in the vegetative cells, irrespective of the differences in the timing and the mode of PM II and the behavior of the organelle nucleoids in generative/sperm cells.

\section{Materials and methods}

\section{Plant materials and observation of mature pollen by the squash method}

Lilium longiflorum, a generous gift from S. Matsuo, was grown on soil under greenhouse conditions in S. Matsuo's field in Tomisato, Chiba, Japan. Pharbitis nil, Silene latifolia, Pelargonium zonale and Plumbago auriculata were grown on soil under greenhouse conditions.

Epifluorescence microscopic observation of mature pollen by the squash method was performed according to the method described previously (Kuroiwa and Suzuki 1980, Saito 1997). In brief, anthers excised from the flower buds just before anthesis were excised on a glass slide in a fixative solution of $2 \%$ glutaraldehyde in TAN buffer $(20 \mathrm{mM}$ Tris- $\mathrm{HCl}, \mathrm{pH} 7.6,0.5 \mathrm{mM}$ EDTA, $7 \mathrm{mM} 2$-mercaptoethanol and $1.2 \mathrm{mM}$ spermidine). The fixed sample $(5 \mu \mathrm{l})$ was mixed with $5 \mu \mathrm{l}$ of a solution of 4',6-diamidino-2-phenylindole (DAPI) at $1 \mu \mathrm{g} / \mathrm{ml}$ in TAN buffer and $1 \mathrm{mg} / \mathrm{ml} n$-propyl gallate dissolved in $50 \%$ glycerol, covered with a coverslip, and then squashed by applying gentle pressure to the coverslip. After blotting the excess liquid with filter paper, the sample was observed with an epifluorescence microscope (BHS-RFC; Olympus, Tokyo, Japan; Kuroiwa et al. 1986, 1990). Photographs were taken at magnifications of $\times 66, \times 132, \times 250, \times 330$ and $\times 1500$ on $35 \mathrm{~mm}$ Neopan 400 PRESTO film (Fuji Photo Film Ltd., Tokyo, Japan).

\section{Fixation and embedding samples in Technovit 7100 resin}

Anthers were collected from buds 0 to 3 days before anthesis and fixed for $12 \mathrm{hr}$ at $4{ }^{\circ} \mathrm{C}$ in $4 \%$ paraformaldehyde that was buffered with $20 \mathrm{mM}$ sodium cacodylate at $\mathrm{pH}$ 7.5. The fixed samples were dehydrated in an ethanol series, and embedded in Technovit 7100 resin (Kulzer and Co., Wehrheim, Germany) according to the method described by Kuroiwa et al. (1991). Thin sections $(0.6 \mu \mathrm{m})$ were cut with a glass knife on an ultramicrotome (MT-7; RMC EIKO, Kawasaki, Japan or ULTRACUT UCT; Leica, Wien, Austria). The sections were placed in a drop of distilled water on a 
coverslip coated with aminopropyltriethoxysilane and glutaraldehyde (Gottlieb and Glaser 1975) and air-dried (Kuroiwa et al. 1991).

\section{In situ hybridization}

In situ hybridization using digoxygenin (DIG)-labeled RNA probes was performed as described previously (Fujie et al. 1995). In brief, a $3.8 \mathrm{kbp}$ (base pair) nuclear genomic fragment of the $18 \mathrm{~S} / 25 \mathrm{~S}$ rRNA gene from Oryza sativa was cloned into pBluescript SKII+. DIG-labeled sense (for negative control) and antisense RNA probes were prepared using DIG-11-UTP in an in vitro transcription reaction according to the manufacturer's protocol (Boehringer Mannheim Biochemicals, FRG). The sections attached to the coverslips were pre-hybridized in hybridization buffer containing $5 \times$ Denhardt's solution, $0.75 \mathrm{M} \mathrm{NaCl}, 5 \mathrm{mM}$ EDTA, $1 \mathrm{mg} / \mathrm{ml}$ salmon DNA, $50 \mathrm{mM}$ sodium phosphate ( $\mathrm{pH} 7.2$ ) and $50 \%$ formamide (McFadden 1990). After incubation at $25^{\circ} \mathrm{C}$ for $30 \mathrm{~min}$, the DIG-labeled probes were added to the hybridization solution (at a final concentration of $1-10 \mu \mathrm{g} / \mathrm{ml})$ and incubated for $12 \mathrm{hr}$ at $45^{\circ} \mathrm{C}$. The coverslips with the thin sections were washed in $4 \times \mathrm{SSC}$ containing $0.1 \% \mathrm{SDS}(30 \mathrm{~min}$ at room temperature), $1 \times \mathrm{SSC}$ containing $0.1 \% \mathrm{SDS}(30 \mathrm{~min}$ at $53^{\circ} \mathrm{C}$ twice $), 0.1 \times \mathrm{SSC}$ containing $0.1 \% \mathrm{SDS}\left(30 \mathrm{~min}\right.$ at $\left.53^{\circ} \mathrm{C}\right)$ and $\mathrm{PBS}(137 \mathrm{mM} \mathrm{NaCl}, 2.7 \mathrm{mM}$ $\mathrm{KCl}, 4.3 \mathrm{mM} \mathrm{Na} \mathrm{HPO}_{4}, 1.4 \mathrm{mM} \mathrm{K \textrm {KH } _ { 2 }} \mathrm{PO}_{4}, 10 \mathrm{~min}$ at room temperature, three times). Then the probes were detected with anti-DIG antibodies (Boehringer Mannheim Biochemicals, FRG) and fluorescein isothiocyanate (FITC) conjugated second antibodies (anti-mouse-immunoglobulin goat antibody conjugated with FITC, TAGO, Inc., Burlingame, CA, USA). The samples were mounted with $1 \mu \mathrm{g} / \mathrm{ml}$ DAPI dissolved in TAN buffer and $1 \mathrm{mg} / \mathrm{ml} n$-propyl gallate dissolved in $50 \%$ glycerol, and observed with an epifluorescence microscope. Photographs were taken as described above.

\section{Results}

\section{Characterization of pollen type}

Images of mature pollen grains of Lilium longiflorum, Pharbitis nil, Silene latifolia, Pelargonium zonale and Plumbago auriculata examined by DAPI-fluorescence microscopy using the squash method are shown in Fig. 1. The mature pollen of L. longiflorum (Fig. 1A, B) and P. nil (Fig. 1C, D) were bicellular, indicating that PM II was not yet complete in the mature pollen grain. In the mature pollen of L. longiflorum, DAPI-fluorescent spots were not observed in the vicinity of the generative nucleus (Fig. 1B), indicating the absence of organelle nucleoids in the generative cell. In the mature pollen of $P$. nil, DAPI-fluorescent spots were observed in the generative cell (Fig. 1D), indicating the presence of organelle nucleoids. The mature pollen of $S$. latifolia (Fig. 1E, F), P. zonale (Fig. $1 \mathrm{G}, \mathrm{H}$ ) and P. auriculata (Fig. 1I, J) was tricellular, indicating that PM II had already finished. In $S$. latifolia, DAPI-fluorescent spots were not observed in the vicinity of the sperm nucleus (Fig. 1F). In contrast, in $P$. zonale, many organelle nucleoids were observed in the cytoplasm of both sperm cells as tiny fluorescent spots (Fig. 1H). Two types of organelle nucleoids were distinguished in the mature sperm cells of $P$. auriculata pollen (Fig. 1J); the DAPI fluorescence of one type was stronger than that of the other. These nucleoids were identified as plastid-nucleoids and mitochondrial-nucleoids, respectively (Saito et al. manuscript in preparation).

Accumulation of rRNA in the generative cells of mature pollen grains of L. longiflorum and P. nil

To compare the density of cytoplasmic rRNA in the generative and vegetative cells, in situ hybridization for $18 \mathrm{~S} / 25 \mathrm{~S}$ rRNA was performed in L. longiflorum and P. nil. Fig. 2 shows sections of a mature pollen grain hybridized with antisense probes for rRNA genes. DAPI fluorescence microscopy revealed the absence of organelle nucleoids in the generative cell of L. longiflorum (Fig. 2A), while some organelle nucleoids in the generative cell of $P$. nil (Fig. 2C). In both plant species, hybridization signals for rRNA were more intense in the generative cell than in the vegetative cell 

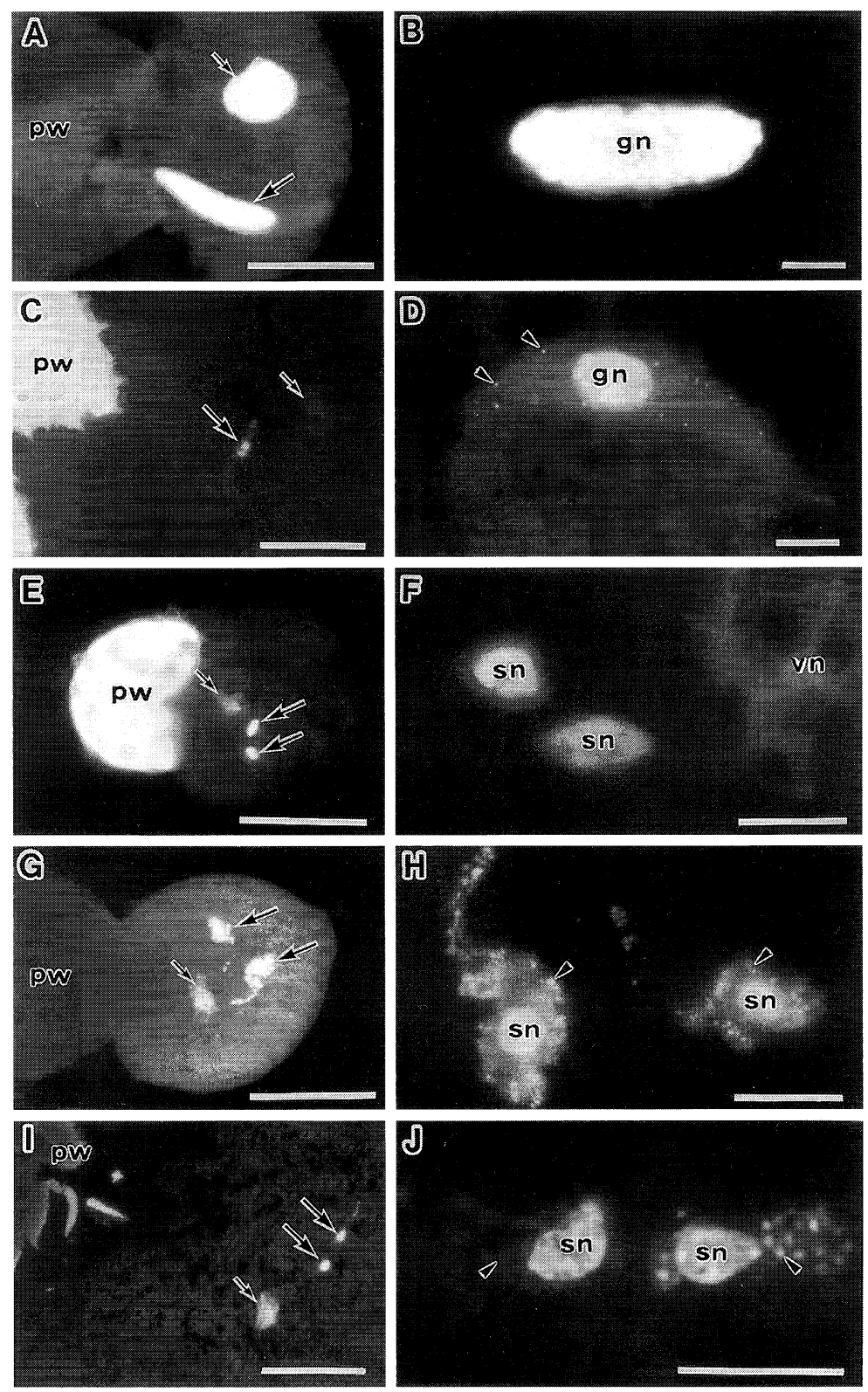

Fig. 1. DAPI-fluorescence images of mature pollen grains of Lilium longiflorum (A and B), Pharbitis nil ( $\mathrm{C}$ and D), Silene latifolia (E and F), Pelargonium zonale ( $\mathrm{G}$ and $\mathrm{H})$ and Plumbago auriculata (I and J) observed by the squash method. Cell nuclei emitted bluish-white fluorescence. Pollen walls emit strong auto-fluorescence (A, C, E, G and I). Large arrows, generative/sperm cells; small arrows, vegetative nuclei; pw, pollen wall; gn, generative nucleus; arrowheads, organelle nucleoids. Bars in A, C, E, G and I represent $50 \mu \mathrm{m}$ and bars in B, D, F, H and $\mathrm{J}$ represent $10 \mu \mathrm{m}$.

(Fig. 2B, D), indicating that there was more rRNA in the generative cells of the mature bicellular pollen of L. longiflorum and P. nil.

Presence of rRNA in the sperm cells of mature pollen grains of S. latifolia, P. zonale and P. auriculata

To determine whether rRNA remained at high density in the sperm cells after PM II, in situ 

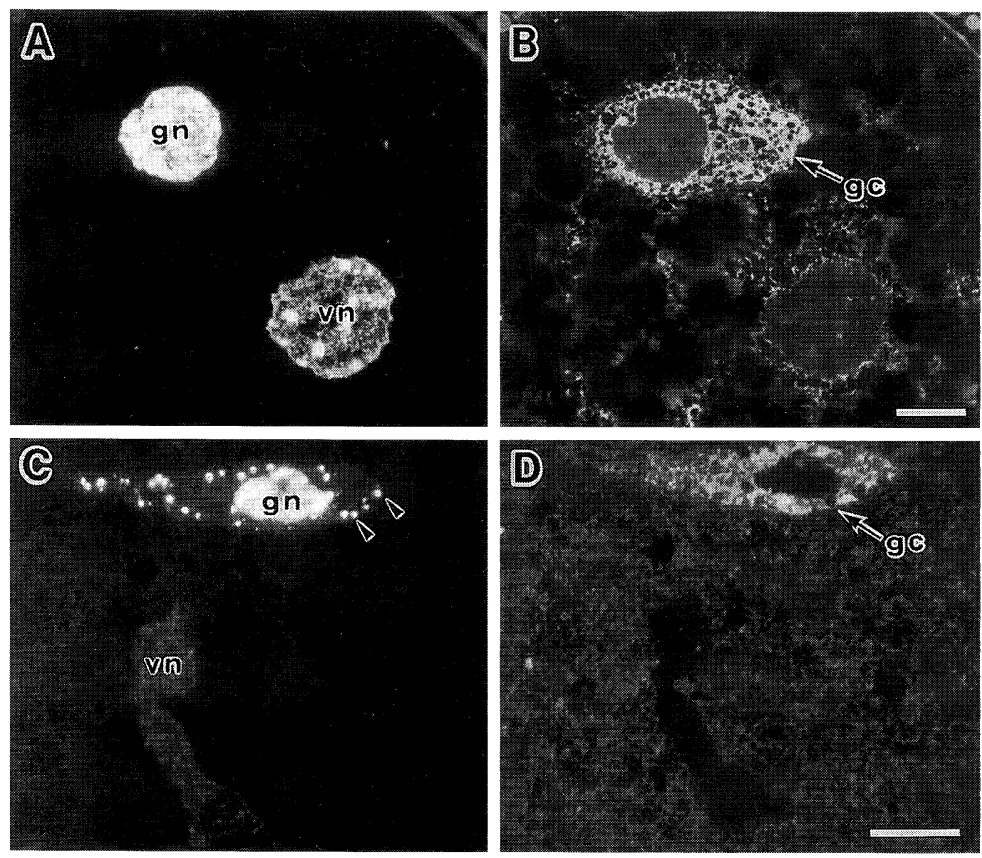

Fig. 2. Epifluorescence micrographs of cytoplasmic rRNA visualized by in situ hybridization on sections of mature pollen of $L$. longiflorum and $P$. nil. DAPI image (A and C) shows vegetative and generative nuclei and organelle nucleoids. Localization of cytoplasmic rRNA was visualized by in situ hybridization (B and D). gc, generative cell; gn, generative nucleus; vn, vegetative nucleus; arrowheads, organelle nucleoids. Bars represent $10 \mu \mathrm{m}$.

hybridization was also performed in the mature tricellular pollen of S. lutifolia, P. zonale and P. auriculata. Fig. 3 shows sections of mature pollen grains hybridized with antisense probes for rRNA genes. No organelle nucleoids were present in the sperm cells of S. latifolia (Fig. 3A), while organelle nucleoids were observed in the sperm cells of $P$. zonale (Fig. 3C) and P. auriculata (Fig. $3 \mathrm{E})$. In all three plant species, the rRNA hybridization signals in the sperm cells were at least as intense as those in the cytoplasm of the vegetative cells (Fig. 3B, D, F), indicating that the density rRNA in the sperm cells was the same or more than in the vegetative cells in mature tricellular pollen of S. latifolia, P. zonale and P. auriculata.

\section{Discussion}

In a previous study, we reported that the amount of rRNA in the generative cell is as high as that in the vegetative cell in mature pollen of Nicotiana tabacum (Saito et al. 1998). In the present study, similar results were also obtained for five plant species, Lilium longiflorum, Pharbitis nil, Silene latifolia, Pelargonium zonale and Plumbago auriculata. Moreover, in three of these species ( $L$. longiforum, $P$. nil and P. zonale), there was significantly dense rRNA in the generative/sperm cells than in the vegetative cell. The presence of rRNA at high density in generative/sperm cells suggests that these cells have the potential for gene expression and this seems to be a general characteristic of pollen grains in plants.

Why are there different densities of rRNA in vegetative and generative/sperm cells? Changes in the cell volume may affect the density of rRNA. The volume of the vegetative cell increases during pollen maturation after PM I, whereas that of the generative cell does not. The very rapid increase in vegetative cell volume may dilute the density of rRNA. However, the continuous synthesis of rRNA in generative/sperm cells may also contribute to the differences in the density of rRNA in 

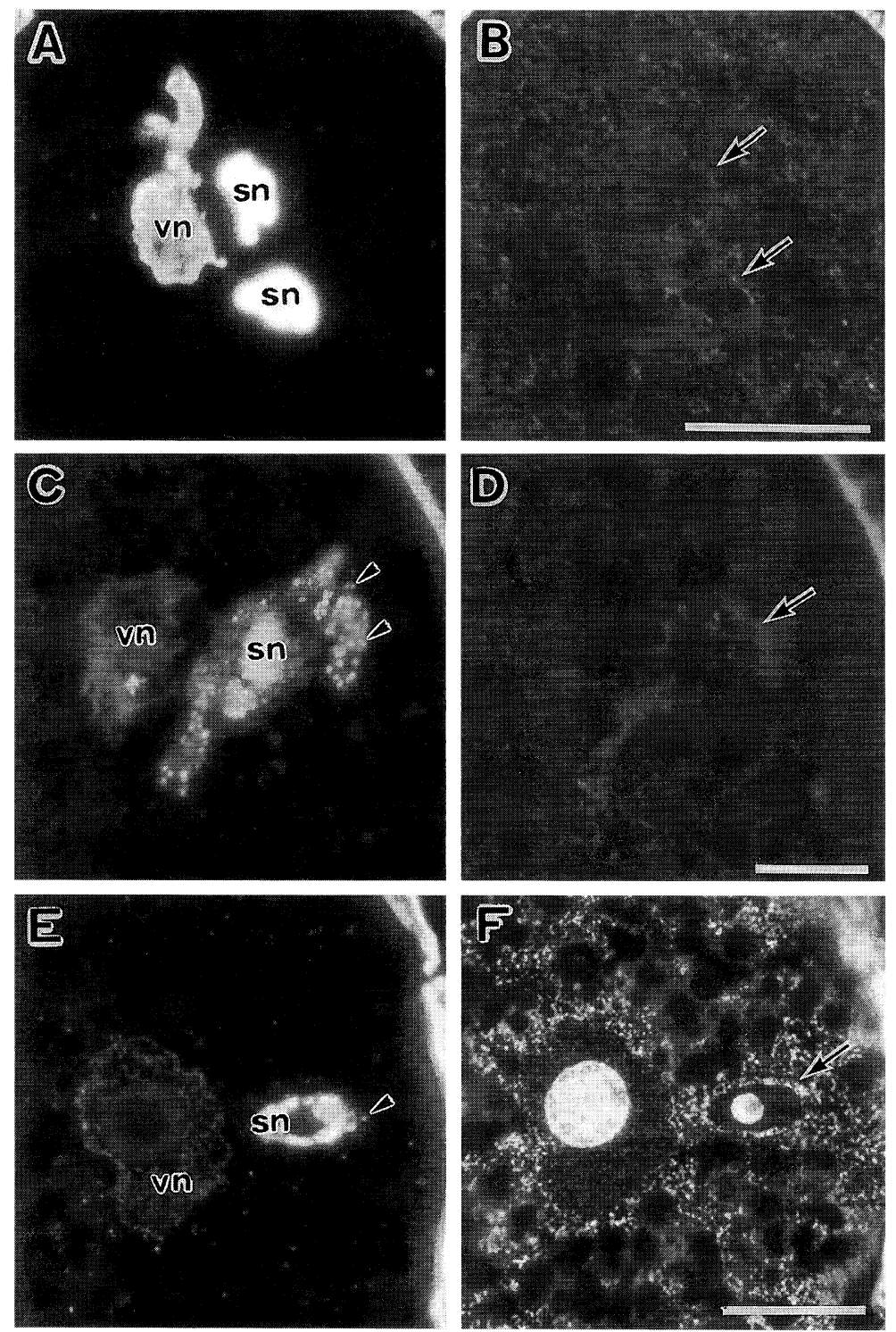

Fig. 3. Epifluorescence micrographs of cytoplasmic rRNA visualized by in situ hybridization on sections of mature pollen of Silene latifolia, P. zonale and P. auriculata. DAPI image (A, C and E) shows vegetative and generative nuclei and organelle nucleoids. Localization of cytoplasmic rRNA was visualized by in situ hybridization (B, D and F). sn, sperm nucleus; vn, vegetative nucleus. Arrows, sperm cells; arrowheads, organelle nucleoids. Bars represent $10 \mu \mathrm{m}$.

the two types of cells. The presence of small but conspicuous nucleoli in generative cells during the maturation of tobacco pollen (Saito et al. 1997) supports this hypothesis. Ultimately, successive examination of the volume and rRNA density of the generative/sperm cells during pollen development will be necessary to determine whether rRNA is synthesized in the generative/sperm cells.

The presence of a high density of rRNA (and ribosomes) implies that generative/sperm cells have the potential for gene expression. It has been reported that in germinating pollen tubes generative/sperm cells incorporate labeled precursors for the synthesis of RNA and protein (Reynolds and Raghavan 1982, Haskell and Rogers 1985, Mascarenhas et al. 1969), which is in agreement with our hypothesis. However, we presently do not know what gene products are synthesized on the ribo- 
somes accumulating at high density in the cytoplasm of generative/sperm cells. As generative cells must undergo PM II, one may suppose that there is a high density of rRNA in the generative cells to prepare for the synthesis of the proteins involved in PM II. However, the presence of rRNA in the sperm cells of tricellular pollen, which have finished PM II, indicates that rRNA does not exist only for PM II.

On the other hand, rRNA was present at high density in the generative/sperm cells irrespective of the presence or absence of organelle nucleoids in the cells. This also indicates that rRNA in these does not exist just for the degradation or preservation of organelle nucleoids, which has been considered a key step to determining the pattern of organelle inheritance (Kuroiwa 1991, Mogensen 1996). These results indicate that ribosomes accumulating in the cytoplasm of generative/sperm cells may be involved in housekeeping events, which may occur after PM II. Alternatively, various post-PM II events, such as the movement of generative/sperm cells in the pollen tube or the process of fertilization, may require a large number of ribosomes in sperm cells. This possibility should be examined by observing changes in the density of rRNA in the generative/sperm cells after pollen tube germination.

At present, no genes expressed specifically in generative/sperm cells have been isolated (Tanaka 1997), although the generative cell-specific proteins from L. longiflorum (Ueda et al. 1994, 1995) are the most probable candidate products of genes expressed specifically in generative/sperm cells. The occurrence of transcription in the generative/sperm cell nucleus (Haskell and Rogers 1985, Reynolds and Raghavan 1982) and the presence of ribosomes at a high density in the cytoplasm of generative/sperm cells indicate that the potential for gene expression is preserved in generative/sperm cells. However, this does not necessarily imply the presence of generative/sperm cellspecific gene expression. Nonetheless, our results support the hypothesis that generative/sperm cellspecific gene expression is possible, and this is worth exploring.

\section{Acknowledgments}

The authors thank Associate Professor S. Kawano, Dr. Sodmergen and Dr. T. Takano for technical advice and helpful discussion. They also thank Mr. S. Matsuo for his generous gift of the Lilium longiflorum plants. This research was supported by a grant for Special Research on Priority Areas (to T. K., project no. 06101002) from the Ministry of Education, Science and Culture of Japan, and by a grant for a Pioneering Research Project in Biotechnology from the Ministry of Agriculture, Forestry and Fisheries of Japan.

\section{References}

Bedinger, P. 1992. The remarkable biology of pollen. Plant Cell 4: 879-887.

Fujie, M., Kuroiwa, H., Kawano, S. and Kuroiwa, T. 1995. Changes in cytoplasmic rRNA density in the root apical meristem observed by high reslution in situ hybridization. Cytologia 60: 149-158.

Gottlieb, D. I. and Glaser, L. 1975. A novel assay of neuronal cell adhesion. Biochem. Biophys. Res. Comm. 64: 815-821.

Haskell, D. W. and Rogers, O. M. 1985. RNA synthesis by vegetative and sperm nuclei of trinucleate pollen. Cytologia 50: 805-809.

Horvitz, H. R. and Herskowitz, I. 1992. Mechanisms of asymmetric cell division: Two Bs or not two Bs, that is the question. Cell 68: 237-255.

Kuroiwa, T. 1991. The reprication, differentiation, and inheritance of plastids with emphasis on the concept of organelle nuclei. Int. Rev. Cytol. 128: 1-62.

— and Suzuki, T. 1980. An improved method for the demonstration of the in situ chloroplast nuclei in higher plants. Cell Struc. Func. 5: 195-197.

—, Miyamura, S., Kawano, S., Hizume, M., Toh-e, A., Miyakawa, I. and Sando, N. 1986. Cytological characterization of NOR in the bivalent of Saccharomyces cerevisiae. Exp. Cell Res. 165: 199-206.

-, Kuroiwa, H., Mita, T. and Fujie, M. 1990. Fluorescence microscopic study of the formation of giant mitochondrial nu- 
clei in the young ovules of Pelargonium zonale. Protoplasma 158: 191-194.

-, Fujie, M., Mita, T. and Kuroiwa, H. 1991. Application of embedding of samples in Technovit 7100 resin to observations of small amounts of DNA in cellular organelles associated with cytoplasmic inheritance. Appl. Fluoresce. Technol. 3: 23-25.

LaFountain, Jr. J. R. and LaFountain, K. L. 1973. Comparison of density of nuclear pores in vegetative and generative nuclei in pollen of Tradencantia. Exp. Cell Res. 78: 472-476.

Mascarenhas, J. P. and Bell, E. 1969. Protein synthesis during germination of pollen studies on polyribosome formation. Biochem. Biophys. Acta 179: 199-203.

McCormick, S. 1993. Male gametophyte development. Plant Cell 5: 1265-1275.

McFadden, G. I. 1990. Evidence that cryptomonad chloroplasts evolved from photosynthetic eukaryotic endosymbionts. J. Cell Sci. 95: 303-308.

Mogensen, H. L. 1996. The hows and whys of cytoplasmic inheritance in seed plants. Amer. J. Bot. 83(3): 383-404.

Reynolds, T. L. and Raghavan, V. 1982. An autoradiographic study of RNA synthesis during maturation and germination of pollen grains of Hyoscyamus niger. Protoplasma 111: 177-188.

Russell, S. D. 1984. Ultrastructure of the sperm of Plumbago zeylanica. Planta 162: 385-391.

-, Strout, G. W., Stramski, A. K., Mislan, T. W., Thompson, R. A. and Schoemann, L. M. 1996. Microgametogenesis in Plumbago zeylanica (Plumbaginaceae). 1. Descriptive cytology and three-dimensional organization. Amer. J. Bot. 83(11): 1435-1453.

Saito, C., Fujie, M., Sakai, A., Kuroiwa, H. and Kuroiwa, T. 1997. Extent of the condensation of nuclear chromatin and the localization of RNA during pollen development in Nicotiana tabacum. Cytologia 62(2): 121-132.

,,,---- and - 1998. Detection and quantification of rRNA by high-resolution in situ hybridization in pollen grains. J. Plant Res. 111: 45-52.

Sodmergen, Chen, G. H., Hu, Z. M., Guo, F. L. and Guan, X. L. 1995. Male gametophyte development in Plumbago zeylanica: cytoplasm localization and cell determination in the early generative cell. Protoplasma 186: 79-86.

Tanaka, I. 1997. Differentiation of generative and vegetative cells in angiosperm pollen. Sex. Plant Reprod. 10(1): 1-7.

Terasaka, O. and Tanaka, R. 1974. Cytological studies on the nuclear differentiation in microspore division of some angiosperms. Bot. Mag. Tokyo 87: 200-217.

Twell, D., Patel, S., Sorensen, A., Roberts, M., Scott, R., Draper, J. and Foster, G. 1993. Activation and developmental regulation of an Arabidopsis anther-specific promotor in microspores and pollen of Nicotiana tabacum. Sex. Plant Reprod. 6: $217-224$.

Ueda, K. and Tanaka, I. 1994. The basic proteins of male gametic nuclei isolated from pollen grains of Lilium longiflorum. Planta 192: 446-452.

— and - 1995. The apprarance of male gamete-specific histones gH2B and gH3 during pollen development in Lilium longiflorum. Dev. Biol. 169: 210-217.

Wagner, V. T., Cresti, M., Salvatici, P. and Tiezzi, A. 1990. Changes in volume, surface area, and frequency of nuclear pores on the vegetative nucleus of tobacco pollen in fresh, hydrated, and activated conditions. Planta 181: 304-309. 